\title{
Continuous Non-invasive Hemoglobin Monitoring during Orthopedic Surgery: A Randomized Trial
}

\author{
Jesse M Ehrenfeld ${ }^{1 *}$, Justin P Henneman ${ }^{2}$, Catherine M Bulka ${ }^{1}$ and Warren S Sandberg ${ }^{1}$
}

${ }^{1}$ Vanderbilt University, USA

${ }^{2}$ North Shore, LIJ School of Medicine at Hofstra University, USA

\begin{abstract}
Blood transfusions during orthopedic surgery increase the risk of adverse outcomes and are costly. In current practice, laboratory hemoglobin values are used to determine the need for blood transfusion, but testing is intermittent. We hypothesized that continuous non-invasive hemoglobin monitoring (SpHb) could reduce intraoperative blood transfusions. Patients undergoing elective orthopedic surgery were randomized to receive standard care alone or standard care with SpHb monitoring. Of the 327 patients enrolled (170 intervention, 157 control), $0.6 \%$ received intraoperative transfusions in the intervention group compared to $4.5 \%$ in the control group, for an absolute risk reduction of $4 \%(95 \% \mathrm{Cl}:-7 \%$ to $-0.4 \%)$. The amount of red blood cell units transfused did not differ between the groups, nor did the rate of laboratory hemoglobin testing. The use of continuous noninvasive hemoglobin monitoring may reduce the rate of transfusions when compared to standard care using intermittent laboratory hemoglobin testing.
\end{abstract}

Keywords: Intraoperative monitoring; Blood transfusions; Orthopedic surgery

\section{Introduction}

When available, Hemoglobin concentration $(\mathrm{Hb})$ values are used as a primary indicator of need for red blood cell transfusion [1]. Laboratory $\mathrm{Hb}$ determination requires provider directed blood sample collection and significant time to process - delaying the receipt of the laboratory result from when the clinical need for $\mathrm{Hb}$ measurement is established [2,3]. This means that during surgery, initial and subsequent transfusion decisions may be made without recent $\mathrm{Hb}$ results. Not surprisingly, inadequate information about a patient's circulating hemoglobin is strongly associated with inappropriate transfusions [4]. Since blood transfusions are associated with postoperative infection, cancer recurrence, length of stay, and mortality, in addition to being costly, continued efforts to reduce unnecessary transfusions are warranted [5-10].

Recent advancements have made continuous and non-invasive hemoglobin monitoring $(\mathrm{SpHb})$ possible through multiwavelength Pulse CO-Oximetry [11]. Several studies have indicated that $\mathrm{SpHb}$ provides values comparable to laboratory $\mathrm{Hb}$ for both absolute and changes in hemoglobin values, while others have suggested $\mathrm{SpHb}$ is unreliable and correlates poorly with laboratory $\mathrm{Hb}$ [12-21]. In spite of its limitations, continuous $\mathrm{SpHb}$ monitoring may provide useful real-time information to the anesthesiologist during the transfusion decision making process, perhaps by making $\mathrm{Hb}$ trend data visible. We hypothesized that $\mathrm{SpHb}$ monitoring could reduce both the rate of intraoperative blood transfusions and the amount of red blood cell units transfused.

\section{Materials and Methods}

The study setting was a large tertiary care center (Massachusetts General Hospital, Boston, MA). The study (\#2009P002600) was approved by the Partners Healthcare Human Research Committee (IRB). Designed to be a 6-month pilot study, subjects were screened for eligibility and enrolled between February and July of 2010. An a priori power analysis was not performed, as we did not have a reasonable estimate of the effect size prior to launching the pilot study. Adult patients (age $\geq 18$ years) were eligible if they were undergoing elective orthopedic surgery under general anesthesia. There were no exclusion criteria.

After study enrolment, randomization to receive either standard care alone (Standard Care Group) or standard care with SpHb monitoring (SpHb Group) was conducted for this parallel-group study using block randomization with a block size of 4 . All orthopedic cases were randomized without regard for procedure type. Case demographics and clinical data were obtained from the electronic intraoperative record. Demographic data included American Society of Anesthesiologists (ASA) physical status classification, age, and gender. Clinical data included procedure, surgical duration, estimated blood loss, $\mathrm{Hb}$ values, and intraoperative transfusions. We also collected information (date, time, number of units transfused) about transfusion, for both the initial postoperative period (defined as twenty-four hours post-surgical end time) and the entire hospitalization. Intraoperative and postoperative blood transfusions were considered to be transfusions of packed red blood cells or whole blood, but not other blood products such as fresh frozen plasma or platelets.

Laboratory $\mathrm{Hb}$ was obtained by venous or arterial blood samples taken at the discretion and direction of the patient's anesthesiologist. Because the focus of our study was on understanding if continuous monitoring of hemoglobin during surgery could reduce the rate of red blood cell transfusions, rather than the accuracy of the SpHb device, we did not dictate as a part of the study protocol intraoperative hemoglobin values. When available however, samples were analyzed in the hospital's central laboratory with a Siemens Rapidlab 1265 calibrated

*Corresponding author: Jesse M. Ehrenfeld, Vanderbilt University, 1301 Medical Center Drive, Suite 4648, Nashville, TN 37212, USA, Tel: 615-936-5194; Fax: 615-936-6493; E-mail: jesse.ehrenfeld@vanderbilt.edu

Received August 01, 2014; Accepted October 29, 2014; Published November 20,2014

Citation: Ehrenfeld JM, Henneman JP, Bulka CM, Sandberg WS (2014) Continuous Non-invasive Hemoglobin Monitoring during Orthopedic Surgery: A Randomized Trial. J Blood Disorders Transf 5: 237. doi: 10.4172/2155-9864.1000237

Copyright: (c) 2014 Ehrenfeld JM, et al. This is an open-access article distributed under the terms of the Creative Commons Attribution License, which permits unrestricted use, distribution, and reproduction in any medium, provided the original author and source are credited. 


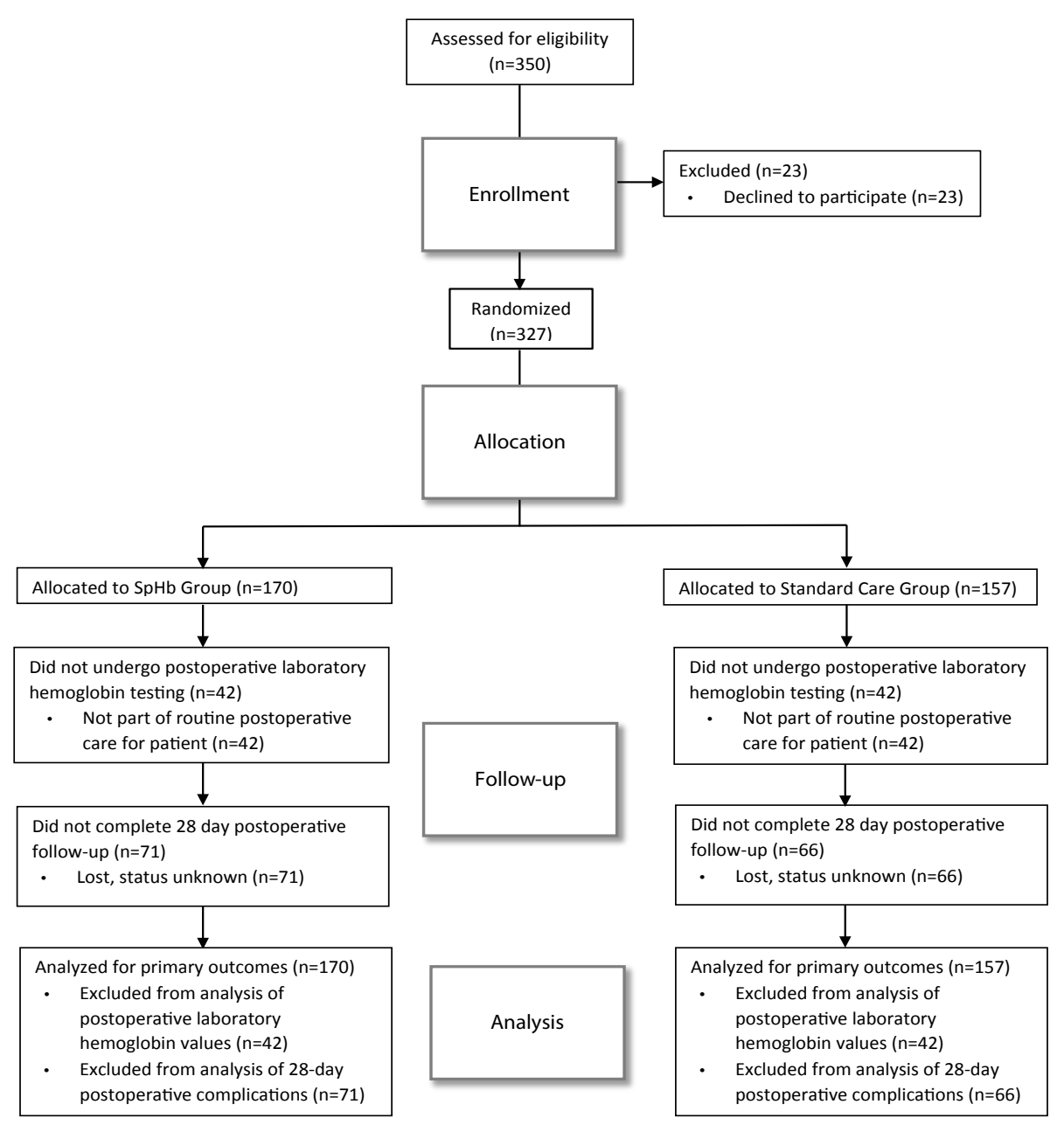

Figure 1: Flow diagram of pilot randomized controlled trial comparing standard care with continuous hemoglobin monitoring during surgery.

to manufacturer's specifications. Records were obtained through an electronic database query of the hospital clinical data repository to obtain preoperative, intraoperative, and postoperative hemoglobin values. Preoperative $\mathrm{Hb}$ values were defined as the most recently available laboratory $\mathrm{Hb}$ results prior to entering the operating room; postoperative $\mathrm{Hb}$ values were defined as the first available laboratory $\mathrm{Hb}$ result after the patient left the operating room.

$\mathrm{SpHb}$ values were obtained with a multiwavelength Pulse COOximeter and adhesive sensor (Radical-7 Monitor and Rainbow Resposable Adhesive Sensor, Rev E, Masimo, Irvine, CA). Pulse CO-Oximetry technology utilizes multiple wavelengths of light with advanced signal processing and adaptive filters to identify, isolate, and quantify blood constituents including total hemoglobin, methemoglobin, and carboxyhemobin, along with oxygen saturation $\left(\mathrm{SpO}_{2}\right)$, pulse rate, and perfusion index. The $\mathrm{SpHb}$ monitor was mounted in the operating room on the anesthesia machine in a readily accessible and visible position for the anesthesia care team to view. The anesthesia care provider was informed of the device and its proper use. The $\mathrm{SpHb}$ monitors displayed and recorded $\mathrm{SpHb}$ and other measurements continuously.

At Massachusetts General Hospital during the study period, there were no formally agreed upon hospital or departmental transfusion guidelines. Decisions about transfusions were made by the anesthesia care team although most practitioners favored a restrictive transfusion approach during the conduct of the study. Anesthesiologists were instructed to care for patients in the Standard Care Group as they normally would. For patients in the SpHb Group, anesthesiologists were instructed to care for patients as they normally would, but to use their own discretion in the use of $\mathrm{SpHb}$ values to help guide need for laboratory $\mathrm{Hb}$ tests and blood transfusions. Notably, there was no attempt to standardize transfusion practices or initiate a specific transfusion protocol during the study period, as we were primarily interested in understanding the impact of adding the $\mathrm{SpHb}$ technology to existing practice.

The primary outcome variables were the frequency of intraoperative red blood cell transfusions and the number of red blood cell units transfused per patient. Secondary outcome variables included the frequency of laboratory $\mathrm{Hb}$ testing and the frequency of postoperative blood transfusions. To monitor safety, each patient also received a follow-up phone call by a trained research assistant 28 days after their surgery to identify the presence of postoperative complications. Patients were asked if they had been to an emergency room or urgent care clinic since their original surgery, and if they had been readmitted to a hospital after their original surgery. Additionally, we screened each patient's medical record to determine if patients had been readmitted 
to the study hospital, had a second surgery, or visited the emergency department at the study hospital.

Concerned that clinicians might alter their transfusion practices solely on the basis of their patient being enrolled in our study, we identified a retrospective cohort (from before the time of the study) to check this possibility. For each patient enrolled in the standard care group $(\mathrm{N}=157)$, we obtained a matched patient from a retrospective cohort taken from the six-month period prior to this study at the same study site. Electronic records were used to identify patients who were matched on three criteria: age, ASA physical status classification, and procedure. We compared the Standard Care Group to the matched retrospective cohort on primary outcomes only, in a set of comparisons planned in advance and separate from the main outcome comparisons between the $\mathrm{SpHb}$ and standard care groups in the active study.

Continuous variables were assessed for normality using histograms. When normally distributed, continuous variables are expressed as mean (SD). Skewed continuous variables are expressed as median (range). Categorical variables are presented as $\mathrm{N}(\%)$. We calculated differences between the groups as the difference between means, medians, or proportions depending on the variable type. These were considered statistically significant when the confidence interval for the difference (95\% CI) did not contain zero.

\section{Results}

We recruited patients from February 2010 through July 2010. A total of 350 patients were screened, and 327 patients were enrolled with 157 patients in the Standard Care Group and 170 patients in the $\mathrm{SpHb}$ Group (Figure 1). For all patients, procedures included primarily hip replacement (33\%), knee replacement (30\%), and spinal surgery (13\%). The retrospective cohort, who received no intervention and was matched to the Standard Care Group, consisted of 157 subjects. As shown in Table 1, baseline characteristics between the Standard Care Group, the SpHb Group, and the matched retrospective cohort were similar. The standard care group was $53.5 \%$ male and had an average age of $61.0 \pm 15.8$ years. The majority $(73.9 \%)$ of the standard care group had an ASA class of 2. The SpHb group was $48.2 \%$ male and had an average age of $61.9 \pm 15.9$ years. Of the 170 patients in this group, 107 (62.9\%) had an ASA class of 2. The mean preoperative laboratory hemoglobin value was $13.5 \pm 1.6 \mathrm{~g} / \mathrm{dL}$ in the SpHb Group and $13.6 \pm 1.5$ $\mathrm{g} / \mathrm{dL}$ in the Standard Care Group.

Table 2 provides information on the primary and secondary outcomes for the Standard Care Group and SpHb Group. The risk difference for intraoperative RBC transfusion between the SpHb Group and the Standard Care Group was -0.04 (95\% CI: -0.07, -0.004). Among the $\mathrm{SpHb}$ Group, only one patient received an intraoperative $\mathrm{RBC}$ transfusion. This patient underwent a spine procedure. The median number of RBC units transfused among both the SpHb Group and the Standard Care Group was 0. We observed no difference between the groups in the amount of estimated blood loss (median difference between groups: 0 ). The likelihood of patients receiving intraoperative $\mathrm{Hb}$ testing was similar in the $\mathrm{SpHb}$ and Standard Care Groups (risk difference: $-0.03,95 \%$ CI: -0.11 to 0.04 ).

No patient from either group received any additional transfusion during the immediate twenty four-hour postoperative period, and only one patient (in the control group) received a transfusion during their entire postoperative hospital course. Of the 327 patients recruited, 190 patients responded to a follow up call (58\%). There were no observed differences at 28 days in the rate of postoperative complications (defined as death, readmission to a hospital, a second surgery, or a visit to the emergency room or an urgent care clinic in the 28 days after surgery) between the SpHb and Standard Care Groups (risk difference: -0.09 , 95\% CI: -0.20 to 0.02). As shown in Table 3, the Standard Care Group had a similar RBC transfusion rate compared to the matched retrospective cohort (risk difference: $0.01,95 \%$ CI: -0.04 to 0.06 ). Median RBC units transfused per case in the Standard Care and the retrospective cohort groups were both 0 .

\section{Discussion}

Our results demonstrate that $\mathrm{SpHb}$-guided blood management in a diverse group of orthopedic surgical is associated with a $4 \%(95 \%$ CI: $-7 \%$ to $-0.4 \%)$ absolute reduction in the risk of having a blood transfusion during surgery compared to standard care. However,

\begin{tabular}{|c|c|c|c|c|c|c|}
\hline & \multicolumn{2}{|c|}{$\begin{array}{l}\text { Retrospective } \\
\text { Cohort (N=157) }\end{array}$} & \multicolumn{2}{|c|}{$\begin{array}{l}\text { SpHb Group } \\
\mathrm{N}=170)\end{array}$} & \multicolumn{2}{|c|}{$\begin{array}{l}\text { Standard Care Group } \\
(\mathrm{N}=157)\end{array}$} \\
\hline & & Total $^{*}$ & & Total $^{*}$ & & Total $^{*}$ \\
\hline Age (years), Mean (SD) & $61.0(15.4)$ & 157 & $61.9(15.9)$ & 170 & $61.0(15.8)$ & 157 \\
\hline \multicolumn{7}{|l|}{ ASA Status, N (\%) } \\
\hline 1 & $10(6.4)$ & 157 & $19(11.1)$ & 170 & $8(5.1)$ & 157 \\
\hline 2 & $111(70.7)$ & 157 & $107(62.9)$ & 170 & $116(73.9)$ & 157 \\
\hline 3 & $35(22.3)$ & 157 & $43(25.2)$ & 170 & $30(19.1)$ & 157 \\
\hline 4 & $1(0.6)$ & 157 & $1(0.6)$ & 170 & $3(1.9)$ & 157 \\
\hline Male, N (\%) & $84(53.5)$ & 157 & $82(48.2)$ & 170 & $84(53.5)$ & 157 \\
\hline Laboratory $\mathrm{Hb}$ value (g/dL), Mean (SD) & - & - & $13.5(1.6)$ & 155 & $13.6(1.5)$ & 147 \\
\hline \multicolumn{7}{|l|}{ Surgical Procedure, N (\%) } \\
\hline Hip Replacement & - & - & $55(32.4)$ & 170 & $52(33.1)$ & 157 \\
\hline Knee Replacement & - & - & $56(32.9)$ & 170 & $42(26.8)$ & 157 \\
\hline Spine Surgery & - & - & $21(12.4)$ & 170 & $22(14.0)$ & 157 \\
\hline Incision and Drainage & - & - & $4(2.4)$ & 170 & $9(5.7)$ & 157 \\
\hline Shoulder Surgery & - & - & $6(3.5)$ & 170 & $8(5.1)$ & 157 \\
\hline Other & - & - & $28(16.5)$ & 170 & $46(29.3)$ & 157 \\
\hline
\end{tabular}

*Total refers to the number of patients for whom relevant data was available (e.g. responded to follow up).

aPreoperative laboratory $\mathrm{Hb}$ values unavailable for the Retrospective Cohort

'Surgical procedure data unavailable for the Retrospective Cohort. Other includes ankle, femur, tibia, Achilles tendon, humerous, elbow, hallux valgus, calcaneous, tibial and wrist repairs; tumor and hardware removal, and leg tendon transplants.

Table 1: Characteristics of patients in the Retrospective Cohort, Standard Care Group, and SpHb Group. 
Page 4 of 5

\begin{tabular}{|c|c|c|c|c|c|c|}
\hline \multirow[b]{2}{*}{ Intraoperative } & \multicolumn{2}{|c|}{$\begin{array}{l}\text { SpHb Group } \\
(\mathrm{N}=170)\end{array}$} & \multicolumn{2}{|c|}{$\begin{array}{c}\text { Standard Care Group } \\
(\mathrm{N}=157)\end{array}$} & \multirow[t]{2}{*}{ Difference $^{a}$} & \multirow[t]{2}{*}{$95 \% \mathrm{Cl}$} \\
\hline & & Total $^{*}$ & & Total* & & \\
\hline Received RBC transfusions, N (\%) & $1(0.6)$ & 170 & $7(4.5)$ & 157 & -0.04 & $(-0.07,-0.004)$ \\
\hline By procedure type, $\mathrm{N}$ & Spine Surgery, 1 & & $\begin{array}{c}\text { Spine Surgery, } 3 \\
\text { Hip Surgery, } 3 \\
\text { Knee Surgery, } 1\end{array}$ & & & \\
\hline RBC units transfused, Median (Range) & $0(0-2)$ & 170 & $0(0-5)$ & 157 & 0.00 & $(0.00,0.00)$ \\
\hline Estimated blood loss (mL), Median (Range) & $100(0-2,000)$ & 170 & $100(0-1,500)$ & 157 & 0.00 & $(0.00,50.00)$ \\
\hline Received laboratory $\mathrm{Hb}$ test, $\mathrm{N}(\%)$ & $18(10.6)$ & 170 & $22(14.0)$ & 157 & -0.03 & $(-0.11,0.04)$ \\
\hline \multicolumn{7}{|l|}{ Postoperative } \\
\hline Received RBC transfusions, N (\%) & $0(0.0)$ & 170 & $1(0.6)$ & 157 & -0.01 & $(-0.02,0.01)$ \\
\hline Laboratory $\mathrm{Hb}$ value (g/dL), Mean (SD) & $11.8(1.5)$ & 128 & $11.7(1.5)$ & 115 & -0.02 & $(-0.40,0.36)$ \\
\hline Suffered complications within 28 days, $N(\%)$ & $15(15.2)$ & 99 & $22(24.2)$ & 91 & -0.09 & $(-0.20,0.02)$ \\
\hline
\end{tabular}

*Total refers to the number of patients for whom relevant data was available (e.g. responded to follow up).

${ }^{a}$ For categorical variables, difference refers to the risk difference. For normally distributed continuous variables, difference refers to the difference in means. For skewed continuous variables, difference refers to the difference in medians.

Table 2: Intraoperative and postoperative outcomes among the Standard Care Group (control) and SpHb Group (intervention).

\begin{tabular}{|c|c|c|c|c|c|c|}
\hline \multirow[b]{2}{*}{ Intraoperative } & \multicolumn{2}{|c|}{$\begin{array}{l}\text { Retrospective Cohort } \\
\qquad(\mathrm{N}=157)\end{array}$} & \multicolumn{2}{|c|}{$\begin{array}{l}\text { Standard Care Group } \\
(\mathrm{N}=157)\end{array}$} & \multirow[t]{2}{*}{ Difference $^{a}$} & \multirow[t]{2}{*}{$95 \% \mathrm{Cl}$} \\
\hline & & Total $^{*}$ & & Total* & & \\
\hline Received RBC transfusions, N (\%) & $9(5.7)$ & 157 & $7(4.5)$ & 157 & 0.01 & $(-0.04,0.06)$ \\
\hline RBC units transfused, Median (Range) & $0(0-3)$ & 157 & $0(0-5)$ & 157 & 0.00 & $(0.00,0.00)$ \\
\hline
\end{tabular}

*Total refers to the number of patients for whom relevant data was available (e.g. responded to follow up).

${ }^{a}$ For categorical variables, difference refers to the risk difference. For normally distributed continuous variables, difference refers to the difference in means. For skewed continuous variables, difference refers to the difference in medians.

Table 3: Intraoperative outcomes among the Standard Care Group (control) and the matched retrospective cohort.

we observed no statistically significant difference in the amount of $\mathrm{RBC}$ units transfused between the two groups. Since patients were randomized to the SpHb and Standard Care Groups, we conclude the difference in the rate of intraoperative transfusions was due to the presence of continuous and non-invasive $\mathrm{Hb}$ values in the SpHb Group. The reduction in intraoperative transfusion rate was not temporary, as evidenced by the absence of postoperative transfusion rates in the $\mathrm{SpHb}$ Group. We believe that the availability of SpHb decreases inappropriate transfusion (either by preventing an initial transfusion, or the transfusion of additional blood products after a single unit has been delivered). To our knowledge, this is the first study to assess the impact of continuous non-invasive hemoglobin monitoring during surgery on the frequency of red blood cell transfusions.

We chose to study orthopedic surgical patients because of their high procedural volume and our desire to assess benefit in patients with a moderate overall risk for transfusion. Thus, if $\mathrm{SpHb}$ monitoring provides clinical and financial benefit in this population, then this result could be robust in patients at higher risk for transfusion. We chose not to create a specific transfusion protocol in either group because we wanted the Standard Care Group to represent real-world behavior at our hospital and also because we wanted to observe the naturally occurring impact of $\mathrm{SpHb}$ monitoring (as distinct from protocol adherence) on clinician behavior.

A small sample size and limited statistical power are obvious limitations of this pilot randomized trial. Another major limitation of our study was the relatively rare event occurrence of a transfusion in the population studied. While a higher risk group (e.g. trauma, cardiac, vascular) may have provided more events for comparison, their case volume would be both dramatically lower, and already heavily biased towards a more apparent clinical indication for transfusion. We sought to capture the population of patients for whom transfusions were a fair possibility, yet where the decision to transfuse was less clear and could be influenced by $\mathrm{Hb}$ monitoring. Although the absolute risk reduction was statistically significant, the low percentage of patients receiving $\mathrm{RBC}$ transfusions may mean that the actual reduction experienced by hospitals could be higher or lower.

A further limitation is that clinicians may have altered their transfusion practices solely on the basis of their patient being enrolled in our study (i.e. Hawthorne effect). We created a retrospective cohort to investigate this possibility, to see if transfusion practices matched historical controls. The transfusion rate and mean RBC units transfused per case in our retrospective cohort group were consistent with the rate of transfusion in our prospective control, the Standard Care Group. This validates that the results achieved in the Standard Care Group did in fact represent standard care for our hospital, and did not represent lower or higher transfusion activity than is typical at our institution for this population. Furthermore, $\mathrm{Hb}$ values were not universally measured in patients, nor were pre and post transfusion $\mathrm{Hb}$ values universally available. Because our institution did not possess transfusion guidelines, it was difficult to assess rationale for transfusion. However, a strong reason for performing the study was the idea that perhaps there are, in fact, robust clinical markers that can help guide our decision-making. Only a small subset, little more than half, of our patients responded to follow-up calls, making postoperative evaluation difficult to generalize.

In the absence of objective information to guide intraoperative transfusion decisions, clinicians can react to readily available indicators that are secondary indicators of circulating red cell mass, such as visual estimation of blood loss or changes in vital signs. While visual estimation of blood loss is commonly performed, its accuracy is limited and should not be used to solely determine need for RBC transfusion [22]. Point of care hemoglobin measurement is also an option, but it is invasive as it requires a blood sample and likely similar in accuracy to $\mathrm{SpHb}[23]$. The lack of continuous $\mathrm{Hb}$ values during surgery may lead to inaccurate assumptions about anemic status, as incomplete knowledge 
of $\mathrm{Hb}$ values is a major factor in inappropriate $\mathrm{RBC}$ transfusions [24,25]. In theory, continuous anemia assessment with $\mathrm{SpHb}$ could guide both the decision to transfuse when hemoglobin values are low or unstable, and the decision to withhold transfusion when $\mathrm{Hb}$ values are not low or unstable. Continuous SpHb monitoring may allow clinicians to more confidently manage patients at lower Hb levels with the knowledge that further drops into a critical anemic range will become readily apparent. Assuming that cardiac output remains roughly constant, continuous $\mathrm{SpHb}$ and $\mathrm{SpO}_{2}$ monitoring gives a rough approximation of oxygen delivery, enabling clinicians to focus more holistically on the patient physiology than on a static $\mathrm{Hb}$ trigger for transfusion.

Given the results of our pilot study, we believe that $\mathrm{SpHb}$ shows promise as an adjunct to current perioperative monitoring practice. However, care should be given to ensure that new technologies are appropriately evaluated in the context of patient care to confirm that the benefits provided outweigh the associated risks and costs.

\section{Acknowledgment}

Source of Funding: Financial support for the conduct of this clinical trial was provided by 5T32GM007592 from the National Institute of Health, as well as by department funds of the Department of Anesthesia, Critical Care, and Pain Medicine, Massachusetts General Hospital. Masimo Corporation provided the devices and sensors used in the study.

\section{References}

1. American Society of Anesthesiologists Task Force on Perioperative Blood Transfusion and Adjuvant Therapies (2006) Practice guidelines for perioperative blood transfusion and adjuvant therapies: an updated report by the American Society of Anesthesiologists Task Force on Perioperative Blood Transfusion and Adjuvant Therapies. Anesthesiology 105: 198-208.

2. Hawkins RC (2007) Laboratory turnaround time. Clin Biochem Rev 28: 179194

3. Dighe AS, Rao A, Coakley AB, Lewandrowski KB (2006) Analysis of laboratory critical value reporting at a large academic medical center. Am J Clin Patho 125: 758-764.

4. Friedman MT, Ebrahim A (2006) Adequacy of physician documentation of red blood cell transfusion and correlation with assessment of transfusion appropriateness. Arch Pathol Lab Med 130: 474-479.

5. Vamvakas EC, Blajchman MA (2009) Transfusion-related mortality: the ongoing risks of allogeneic blood transfusion and the available strategies for their prevention. Blood 113: 3406-3417.

6. Murphy GJ, Reeves BC, Rogers CA, Rizvi SI, Culliford L, et al. (2007) Increased mortality, postoperative morbidity, and cost after red blood cell transfusion in patients having cardiac surgery. Circulation 116: 2544-2552.

7. Popovsky MA, Chaplin HC Jr, Moore SB (1992) Transfusion-related acute lung injury: a neglected, serious complication of hemotherapy. Transfusion 32: 589592.

8. Stainsby D, Jones $H$, Asher D, Atterbury C, Boncinelli A, et al. (2006) Serious hazards of transfusion: a decade of hemovigilance in the UK. Transfus Med Rev 20: 273-282

9. Seeff LB, Hollinger FB, Alter HJ, Wright EC, Cain CM, et al. (2001) Long-term mortality and morbidity of transfusion-associated non-A, non-B, and type $C$ hepatitis: A National Heart, Lung, and Blood Institute collaborative study. Hepatology 33: 455-463.
10. Shander A, Hofmann A, Ozawa S, Theusinger OM, Gombotz H, et al. (2010) Activity-based costs of blood transfusions in surgical patients at four hospitals. Transfusion 50: 753-765

11. Food and Drug Administration K080238-Masimo Rainbow SET Radical-7 Pulse CO-Oximeter. 510(k) Summary.

12. Kim SH, Lilot M, Murphy LS, Sidhu KS, Yu Z, et al. (2014) Accuracy of continuous noninvasive hemoglobin monitoring: a systematic review and metaanalysis. Anesth Analg 119: 332-346.

13. Applegate RL 2nd, Barr SJ, Collier CE, Rook JL, Mangus DB, et al. (2012) Evaluation of pulse cooximetry in patients undergoing abdominal or pelvic surgery. Anesthesiology 116: 65-72.

14. Jung YH, Lee J, Kim HS, Shin SH, Sohn JA, et al. (2013) The efficacy of noninvasive hemoglobin measurement by pulse CO-oximetry in neonates. Pediatr Crit Care Med 14: 70-73.

15. Park YH, Lee JH, Song HG, Byon HJ, Kim HS, et al. (2012) The accuracy of noninvasive hemoglobin monitoring using the radical-7 pulse CO-Oximeter in children undergoing neurosurgery. Anesth Analg 115: 1302-1307.

16. Colquhoun DA, Forkin KT, Durieux ME, Thiele RH (2012) Ability of the Masimo pulse CO-Oximeter to detect changes in hemoglobin. J Clin Monit Comput 26: 69-73.

17. Berkow L, Rotolo S, Mirski E (2011) Continuous noninvasive hemoglobin monitoring during complex spine surgery. Anesth Analg 113: 1396-1402.

18. Miller RD, Ward TA, Shiboski SC, Cohen NH (2011) A comparison of three methods of hemoglobin monitoring in patients undergoing spine surgery. Anesth Analg 112: 858-863.

19. Gayat E, Bodin A, Sportiello C, Boisson M, Dreyfus JF, et al. (2011) Performance evaluation of a noninvasive hemoglobin monitoring device. Ann Emerg Med 57: 330-333

20. Giraud B, Frasca D, Debaene B, Mimoz O (2013) Comparison of haemoglobin measurement methods in the operating theatre. $\mathrm{Br} \mathrm{J}$ Anaesth 111: 946-954.

21. Moore LJ, Wade CE, Vincent L, Podbielski J, Camp E, et al. (2013) Evaluation of noninvasive hemoglobin measurements in trauma patients. Am J Surg 206: 1041-1047.

22. Eipe N PM (2006) Perioperative Blood Loss Assessment - How Accurate? Indian J. Anaesth 50: 35-38.

23. Raikhel M (2012) Accuracy of noninvasive and invasive point-of-care tota blood hemoglobin measurement in an outpatient setting. Postgrad Med 124 250-255.

24. Cook SS, Cangialose CB, Sieburg DM, Kieszak SM, Boudreau R, et al. (1999) Red blood cell transfusions for elective hip and knee arthroplasty: opportunity to improve quality of care and documentation. Clin Perform Qual Health Care 7: 5-16.

25. Tartter PI, Barron DM (1985) Unnecessary blood transfusions in elective colorectal cancer surgery. Transfusion 25: 113-115.
Citation: Ehrenfeld JM, Henneman JP, Bulka CM, Sandberg WS (2014) Continuous Non-invasive Hemoglobin Monitoring during Orthopedic Surgery: A Randomized Trial. J Blood Disorders Transf 5: 237. doi: 10.4172/21559864.1000237 